\title{
Verificación del curva de Laffer, caso Ecuador 2016 - 2017
}

\section{Verification of the Laffer curve, case of Ecuador 2016 - 2017}

\author{
Patricio Esteban Mendieta Andrade ${ }^{1 *}$ \\ ${ }^{1}$ Universidad Católica de Cuenca \\ *pmendietaa@uacue.edu.ec
}

DOI: https://doi.org/10.26871/killkana_social.v2i3.329

\begin{abstract}
Resumen
Es evidente el gran aporte que ha tenido la recaudación tributaria en el Presupuesto General del Estado Ecuatoriano en los últimos años; es por ello, que las autoridades monetarias nacionales han introducido constantemente reformas al marco legal con el afán de optimizar dicho recaudo, a fin de que éste cubra la planificación estatal y se enmarque en la dinámica de crecimiento de la economía nacional. El presente trabajo examina la relación entre la carga tributaria y el ingreso fiscal en nuestro país en los años 2016 y 2017, mediante del reconocimiento empírico de la vigencia de los principios teóricos de la Curva de Laffer, para lo cual se aplicó el análisis estadístico a la serie de datos tanto del PIB, población y niveles de recaudación tributaria obtenidos en las bases de datos de los entes estatales correspondientes, evidenciando, por un lado, el crecimiento de la presión fiscal en el periodo estudiado, y el cumplimiento de los parámetros que establece el modelo teórico; finalmente, se establece la tasa que maximiza el ingreso tributario bajo las especificaciones del modelo econométrico propuesto.
\end{abstract}

Palabras clave: Curva de Laffer, presión tributaria.

\begin{abstract}
It is evident the great contribution that the tax collection has had in the General Budget of the Ecuadorian State in the last years; It is for this reason that the national monetary authorities have constantly introduced reforms to the legal framework with the aim of optimizing this collection, so that it covers the state planning and is part of the growth dynamics of the national economy. This paper examines the relationship between the tax burden and tax revenue in our country in 2016 and 2017, through the empirical recognition of the validity of the theoretical principles of the Laffer curve, for which statistical analysis was applied to the data series of both GDP, population and levels of tax collection obtained in the databases of the corresponding state entities, evidencing, on the one hand, the growth of the fiscal pressure in the period studied, and compliance with the parameters that establishes the theoretical model, although the low significance of its coefficients is noted; finally, the rate that maximizes the tax revenue under the specifications of the proposed econometric model is established.
\end{abstract}

Key words: Laffer curve, tax pressure.

\section{Introducción}

Según informes de los órganos rectores de las finanzas nacionales ecuatorianas el aporte de la recaudación fiscal al Presupuestos General del Estado es cada vez más importante, dada la alta dependencia de los ingresos petroleros y la volatilidad de su precio a nivel mundial; lo cual hace, que los gobiernos de turno utilicen al sistema tributario como una herramienta para abastecerse de recursos y cumplir con sus metas y proyectos planteados, desechando uno de los objetivos básicos de los impuestos, por el cual éstos deberían ser el sistema por el que se garantice un reparto equitativo de la riqueza en una sociedad y se genere un dinamismo económico generalizado a todos los sectores, desarrollando sectores que se han mantenido marginados por la pobreza y sentenciados hoy en día por el paterna- lismo que a la fina es el hijo predilecto de los gobiernos populistas.

Con el antecedente expuesto, en el Ecuador en la década pasada hemos sido testigos de varios cambios al andamiaje legal que sostiene el cobro de tributos en nuestro país, caracterizados por olas de rumores que preceden a cambios que, si bien no han sido substanciales, generan inestabilidad en el sector económico y una imagen denostada a nivel internacional. Por lo expuesto y resaltando la importancia que reviste el estudio de los temas inherentes a la gestión de los tributos en nuestro país, el presente trabajo se plantea analizar la evolución del recaudo fiscal, para lo cual se realizará una evaluación bajo los parámetros establecidos en la Curva de Laffer, en los cuales se explica que un incremento de la presión fiscal no conduce a un aumento efectivo en el ingreso tributario de las naciones. 
El presente documento expone los resultados del trabajo investigativo planteado siendo que como punto posterior a esta introducción se presente el marco teórico en el cual se identifican las particularidades del sistema tributario nacional y las especificaciones del modelo presentado por Laffer; más adelante se presenta la metodología utilizada en este trabajo, las cuales condujeron a que en el punto siguiente se presenten los resultados que sostienen las conclusiones finales.

\section{Marco teórico}

\subsection{Los impuestos en el Ecuador}

A partir de 1979, en el transcurso de los diferentes gobiernos ecuatorianos, se han sucedido una serie de cambios al régimen tributario interno, influenciados bien sea por tendencias conceptuales y técnicas o marcados tintes ideológicos.

Es así que en el Ecuador en 1970 se establece el Impuesto a las Transacciones Mercantiles con una tarifa del $4 \%$; que luego, mediante reforma tributaria implementada por el presidente Dr. Rodrigo Borja, se establece como el Impuesto al Valor agregado (IVA) (Pérez y Vaca, 2015). Se puede anotar que el IVA grava al valor de las transferencias de dominio o a la importación de bienes, derechos de autor, propiedad industrial y otros derechos; además del valor de los servicios prestados (Congreso Nacional, 2004).

Por otro lado, se destaca la importancia del Impuesto a la Renta (IR), el cual "establece el impuesto a la renta global que obtengan las personas naturales, las sucesiones indivisas y las sociedades nacionales o extranjeras (Congreso Nacional, 2004); debiéndose anotar que en el año 2017 la recaudación por éste impuesto representó el $32.83 \%$ de la recaudación total neta del año (SRI, 2018). A lo anotado, el director del Servicio de Rentas Internas, en funciones, Leonardo Orlando, destacó que para el segmento de contribuyentes personas naturales en el 2017 se recaudó 175.2 millones de dólares por este concepto superando a los163.72 millones recaudado en el año 2016 al mismo segmento (Andes, 2018).

En la Tabla 1 se presenta un resumen de los valores recaudados por los distintos impuestos en el Ecuador durante el período 2016 y 2017, siendo importante reconocer que el IVA es el impuesto que más representa en referencia a las recaudaciones totales netas; siendo seguido en importancia, el aporte del IR; por lo que es posible anotar que estos dos tributos son el sostén del sistema tributario nacional. Se advierte un crecimiento interanual del $10.71 \%$ en la recaudación por concepto del IVA y $5.85 \%$ de IR.

Tabla 1 Resumen de recaudación por los diferentes impuestos en el Ecuador año 2016 y 2017

\begin{tabular}{|c|c|c|c|c|c|}
\hline \multirow[b]{2}{*}{ CONCEPTO } & \multicolumn{2}{|c|}{2016} & \multicolumn{2}{|c|}{2017} & \multirow[b]{2}{*}{ Variación interanual } \\
\hline & $\begin{array}{c}\text { Total } \\
\text { recaudado }\end{array}$ & $\begin{array}{c}\text { Análisis } \\
\text { vertical }\end{array}$ & $\begin{array}{c}\text { Total } \\
\text { recaudado }\end{array}$ & $\begin{array}{l}\text { Análisis } \\
\text { vertical }\end{array}$ & \\
\hline Impuesto al Valor Agregado & $5.704 .146,76$ & $45,40 \%$ & $6.314 .931,14$ & $49,63 \%$ & $10,71 \%$ \\
\hline Impuesto a la Renta & $3.946 .284,23$ & $31,41 \%$ & $4.177 .295,15$ & $32,83 \%$ & $5,85 \%$ \\
\hline Impuesto a la Salida de Divisas & $964.658,80$ & $7,68 \%$ & $1.097 .642,48$ & $8,63 \%$ & $13,79 \%$ \\
\hline Impuesto a los Consumos Especiales & $798.329,68$ & $6,35 \%$ & $949.402,13$ & $7,46 \%$ & $18,92 \%$ \\
\hline Impuesto a los Vehículos Motorizados & $194.674,99$ & $1,55 \%$ & $191.480,34$ & $1,50 \%$ & $-1,64 \%$ \\
\hline Impuesto Fomento Ambiental & $140.268,56$ & $1,12 \%$ & $142.123,27$ & $1,12 \%$ & $1,32 \%$ \\
\hline Contribución para la atención integral del cáncer & $90.259,27$ & $0,72 \%$ & $96.677,26$ & $0,76 \%$ & $7,11 \%$ \\
\hline Intereses por Mora Tributaria & $135.665,62$ & $1,08 \%$ & $78.537,92$ & $0,62 \%$ & $-42,11 \%$ \\
\hline $\begin{array}{l}\text { Regalías, patentes y utilidades de conservación mi- } \\
\text { nera }\end{array}$ & $50.210,38$ & $0,40 \%$ & $52.965,13$ & $0,42 \%$ & $5,49 \%$ \\
\hline Multas Tributarias Fiscales & $50.180,96$ & $0,40 \%$ & $49.621,63$ & $0,39 \%$ & $-1,11 \%$ \\
\hline Imp. Activos en el Exterior & $46.910,25$ & $0,37 \%$ & $34.875,98$ & $0,27 \%$ & $-25,65 \%$ \\
\hline RISE & $18.783,20$ & $0,15 \%$ & $22.105,37$ & $0,17 \%$ & $17,69 \%$ \\
\hline Otros Ingresos & $78.554,39$ & $0,63 \%$ & $8.180,26$ & $0,06 \%$ & $-89,59 \%$ \\
\hline Tierras Rurales & $7.853,43$ & $0,06 \%$ & $7.312,81$ & $0,06 \%$ & $-6,88 \%$ \\
\hline $\begin{array}{l}\text { TOTAL RECAUDACIÓN SIN CONTRIBUCIO- } \\
\text { NES SOLIDARIAS }\end{array}$ & $12.226 .780,51$ & $97,30 \%$ & $13.223 .150,87$ & $103,91 \%$ & $8,15 \%$ \\
\hline Contribución $2 \%$ IVA & $402.444,05$ & $3,20 \%$ & $415.113,59$ & $3,26 \%$ & $3,15 \%$ \\
\hline Contribución solidaria sobre las utilidades & $355.239,71$ & $2,83 \%$ & $18.966,17$ & $0,15 \%$ & $-94,66 \%$ \\
\hline Contribución solidaria sobre el patrimonio & $201.539,62$ & $1,60 \%$ & $15.999,20$ & $0,13 \%$ & $-92,06 \%$ \\
\hline Contribución solidaria de un día de remuneración & $60.459,35$ & $0,48 \%$ & $4.852,35$ & $0,04 \%$ & $-91,97 \%$ \\
\hline $\begin{array}{l}\text { Contribución solidaria sobre bienes inmuebles y } \\
\text { derechos representativos de capital de propiedad de } \\
\text { sociedades no residentes }\end{array}$ & $141.064,42$ & $1,12 \%$ & $2.613,75$ & $0,02 \%$ & $-98,15 \%$ \\
\hline $\begin{array}{l}\text { TOTAL RECAUDACIÓN SIN REMISIÓN Y } \\
\text { CON CONTRIBUCIONES SOLIDARIAS(2) }\end{array}$ & $13.387 .527,66$ & $106,55 \%$ & $13.680 .695,93$ & $107,51 \%$ & $2,19 \%$ \\
\hline (-) Notas de crédito y compensaciones & $724.863,00$ & $-5,77 \%$ & $753.681,43$ & $-5,92 \%$ & $3,98 \%$ \\
\hline (-) Devoluciones & $97.827,74$ & $-0,78 \%$ & $201.972,39$ & $-1,59 \%$ & $106,46 \%$ \\
\hline TOTAL NETO & $12.564 .836,92$ & $100,00 \%$ & $12.725 .042,11$ & $100,00 \%$ & $1,28 \%$ \\
\hline
\end{tabular}

Fuente: (SRI, 2018) Estadísticas Generales de Recaudación. Obtenido de https://goo.g1/LKoJtA 
Prosiguiendo con el análisis de la Tabla 1, se destaca como importante el aporte tanto del impuesto a la salida de divisas (ISD) como el gravamen a los consumos especiales (ICE); puntualizando que en el primer caso el hecho generado lo constituye, en términos generales. la transferencia, traslado o envió de divisas al exterior (Presidencia de la República, 2008); mientras el segundo impuesto anotado graba a bienes y servicios concretos tales como tabacos, perfumes, armas, determinados vehículos, servicios de televisión pagada y otros que detalla la Ley Orgánica de Régimen Tributario Interno (LORTI) (Congreso Nacional, 2004).

Durante el período de estudio se aprecia un crecimiento de las recaudaciones sin las contribuciones solidarias, normadas a partir de mediados de 2016 hasta mediados de 2017, de un $8.15 \%$, lo cual consideramos importante si lo comparamos con el crecimiento de la economía ecuatoriana
(PIB) registrado en el año 2017, siendo éste de un $3.0 \%$ en términos reales, explicado por el aumento del gasto del consumo final de los hogares, el gasto de consumo final del Gobierno General y las exportaciones (BCE, 2018). Siendo evidente el resultado de los esfuerzos por parte del ente recaudador fiscal, a fin de incentivar el pago de los impuestos y reducir el sector informal de la economía, ampliando la base de contribuyentes.

Cabe anotar que en el período de análisis se han introducido diferentes cambios en el marco legal tributario, enfocados principalmente a optimizar el sistema, garantizar la progresividad del mismo, incentivar al sector económico y responder a eventualidades que afectaron a la economía ecuatoriana durante el período. De esta manera, en la Tabla 2 se presenta de forma resumida, las principales leyes que entraron en vigencia durante en el período de análisis y los cambios al sistema fiscal introducidos por las mismas.

Tabla 2 Índices de Ajuste del Modelo de Inteligencia emocional

\begin{tabular}{|c|c|c|}
\hline Ley & $\begin{array}{l}\text { Fecha que } \\
\text { entra en } \\
\text { vigencia }\end{array}$ & Principales cambios \\
\hline $\begin{array}{l}\text { Ley Orgánica para el } \\
\text { Equilibrio de las Finan- } \\
\text { zas Públicas }\end{array}$ & $30 / 04 / 2016$ & $\begin{array}{l}\text { - } \quad \text { Reducción de } 4 \text { puntos en el IVA a los consumos don dinero electrónico y } 1 \text { punto en consumos con } \\
\text { tarjetas de crédito o débito. } \\
\text { - Se establece la base imponible máxima para devolución del IVA a personas de tercera edad y } \\
\text { discapacitados en } 2 \text { salarios básicos unificados. } \\
\text { - Se reduce el beneficio a las personas de tercera edad de exonerarse en el IR a únicamente una fracción } \\
\text { básica grabada. } \\
\text { - Cambios en el cálculo del anticipo al IR de las personas naturales obligadas a llevar contabilidad. } \\
\text { - Rebaja en el Anticipo al IR a las empresas que utilicen dinero electrónico. } \\
\text { - } \quad \text { Eliminación del ISD en transacciones físicas de hasta USD } 5.000 \text { al año. } \\
\text { Son exentos del pago del ISD las transferencias al exterior para financiar estudios o gastos por } \\
\text { enfermedades. } \\
\text { En cuanto al ICE, se incrementa a USD 0.16 por unidad, la cerveza industrial a USD } 12.00 \text { por litro } \\
\text { de alcohol, se graba con el } 15 \% \text { a servicios de telefonía fija y planes de telefonía móvil. } \\
\text { Se elimina la exoneración del pago del impuesto ambiental a la contaminación de las personas con } \\
\text { discapacidad (Asamblea Nacional, 2016c). }\end{array}$ \\
\hline $\begin{array}{l}\text { Ley Orgánica de Solida- } \\
\text { ridad y Corresponsabili- } \\
\text { dad por el Terremoto de } \\
16 \text { de abril del } 2016\end{array}$ & $20 / 05 / 2016$ & $\begin{array}{l}\text { - Fija una contribución única sobre las remuneraciones, el patrimonio, las utilidades y los bienes } \\
\text { inmuebles y derechos representativos de capital existentes en el ecuador de propiedad de sociedades } \\
\text { residentes en paraísos fiscales u otras jurisdicciones del exterior } \\
\text { Incrementó la tarifa del IVA a } 14 \% \text { durante un año a partir de la publicación de la ley (Asamblea } \\
\text { Nacional, 2016b) }\end{array}$ \\
\hline $\begin{array}{l}\text { Ley Orgánica para Evitar } \\
\text { la Especulación sobre el } \\
\text { valor de las Tierras y Fi- } \\
\text { jación de Tributos }\end{array}$ & $21 / 07 / 2016$ & $\begin{array}{l}\text { - Graba a la ganancia extraordinaria en la transferencia de dominio de bienes inmuebles tanto rurales } \\
\text { como urbanos y en los aportes de bienes inmuebles a fiedeicomisos y sociedades de carácter } \\
\text { inmobiliario (Asamblea Nacional, 2016c). }\end{array}$ \\
\hline $\begin{array}{l}\text { Ley Orgánica de Incenti- } \\
\text { vos Tributarios para Va- } \\
\text { rios Sectores Productivos } \\
\text { e Interpretativa del Ar- } \\
\text { tículo } 547 \text { del Código Or- } \\
\text { gánico de Organización } \\
\text { Territorial, Autonomía y } \\
\text { Descentralización }\end{array}$ & $12 / 10 / 2016$ & $\begin{array}{l}\text { - } \quad \text { Deducción adicional por gastos de seguros médicos y medicina prepagada. } \\
\text { - } \quad \text { Se fijan tarifas del Impuesto a la Renta Único. } \\
\text { - } \quad \text { Plantea simplicidad en el cálculo del Anticipo al IR para microempresas. } \\
\text { - } \quad \text { Especifica exenciones específicas para la devolución del Anticipo del IR. } \\
\text { PlSD en los pagos de capital o dividendos (Asamblea Nacional, 2016a). }\end{array}$ \\
\hline $\begin{array}{lrr}\text { Ley orgánica para } \\
\text { la reactivación } \\
\text { de la economía, } \\
\text { fortalecimiento } & \text { de } \\
\text { la dolarización y } \\
\text { modernización de la } \\
\text { gestión financiera }\end{array}$ & $29 / 12 / 2017$ & $\begin{array}{l}\text { - } \quad \text { Fija cambios en la tarifa del IR siendo del } 25 \% \text { la tasa general para sociedades y el } 22 \% \text { para } \\
\text { microempresa. } \\
\text { - Limita el beneficio de la reducción de } 10 \text { puntos en el IR para casos de reinversión a exportadores } \\
\text { habituales sociedades manufactureras que cumplen con límites de inclusión laboral y sociedades } \\
\text { dedicadas a turismo receptivo. } \\
\text { - } \quad \text { Modifica el cálculo del anticipo del IR. } \\
\text { - } \quad \text { Incluya la obligatoriedad del uso del sistema financiero para el pago de gastos deducibles. } \\
\text { - } \quad \text { Elimina el Impuesto a la Tierras Rurales (Asamblea Nacional, 2017). }\end{array}$ \\
\hline
\end{tabular}

Nota: Elaboración propia del autor 


\subsection{La Curva de Laffer}

Luego de lo expuesto siempre será latente la pregunta de qué tan eficiente es el sistema tributario de una nación, donde surgen voces que piden reducir los impuestos a fin de estimular el crecimiento económico, confrontados con aquellos que sugieren que los impuestos es la herramienta más eficiente para la redistribución de la riqueza dentro de una sociedad y por lo tanto, es necesario elevar las tasas impositivas a fin de que crezca los recaudos y estos se transfieran a los desfavorecidos por la vía de la inversión gubernamental

Por lo expuesto es importante considerar el término eficiencia tributaria, el cual para Buchanan y Lee (1982) consiste en el equilibrio entre la tasa impositiva y la recaudación y se logra cuando se determinan tipos impositivos tan adecuados que no existe incentivos para cambiarlos, siendo que los contribuyentes se ajustan totalmente a las tasas que se han establecido (Gonzáles y Doria, 2010).

En busca de la anhelada eficiencia tributaria, la Curva de Laffer se constituye como un modelo econométrico que pretende ser una guía en el análisis y representa la relación entre el recaudo por tributos y el tipo impositivo, y se enfoca en demostrar la forma en que afecta a una economía las tasas impositivas, siendo que no necesariamente la subida de impuestos conduce a un incremento en el recaudo (Casparri, 2013).

Basado en los supuestos de Laffer, Wanniski (1978) puntualiza que en el caso hipotético de tener una tasa impositiva del $100 \%$ del ingreso, no existiría producción, dado que todas las rentas obtenidas serían entregadas al Estado, vía impuestos. Siendo que, en el otro extremo de la concepción, con una tasa igual a cero el Estado no tendría ingresos, y su participación en la planteada distribución de la riqueza sería nula (Aliaga y Oropeza, 2015) .

Con este antecedente la Curva de Laffer cumple un papel importante en el medio académico y en el imaginario político desde su aparición en escena en los años 80 durante el gobierno de Reagan en los EEUU, y se ha constituido en el fondo ideal para el debate sobre las tasas de impuestos en la consecución de la eficiencia tributaria en diferentes países. Es así que Varela y Rubiera (2017) confirma la presencia de una relación parabólica como la que presupone el modelo de la Curva de Laffer entre los ingresos fiscales obtenidos del impuesto a la renta de las personas físicas con la tasa impositiva para la economía española; reconociendo una tasa impositiva del $30 \%$ como la que maximizaría los ingresos fiscales; siendo ésta el doble de la tasa impositiva promedio real existente en el período estudiado.

Por su parte, Bejarano (2008) en su estudio verifica que para la economía colombiana en el periodo 19802005, se cumplen los parámetros teóricos especificados en el modelo de la Curva de Laffer, determinando que un ingreso tributario como porcentaje del PIB del $13 \%$ maximiza los recaudos tributarios, concluyendo que las altas tarifas impositivas no generan los resultados esperados para la economía de Colombia, especificando que el nivel impositivo de renta superior al $36 \%$ ubica al sistema en una franja, en la que el aumento de la tasa afecta negativamente en el nivel de ingreso tributario.

Para el mismo caso colombiano, los autores Macías y Cortés (2004) reconoce en su trabajo la transformación de la estructura del sistema fiscal en éste país; dado que los impuestos indirectos, concretamente el IVA, adquieren cada vez mayor relevancia en el total recaudado. De la misma manera, se verifica que las altas tasas cobradas no generan los resultados esperados, más aún se pone en evidencia un crecimiento en los niveles de evasión.

Ya en el plano nacional, la autora Cueva (2012) demuestra en su estudio el cumplimiento de los preceptos teóricos de la Curva de Laffer, en el caso Ecuatoriano, al relacionar la variables ingresos tributarios como porcentaje del PIB en el período 1970-2008; se determina el nivel óptimo de los ingresos reales per cápita corresponden a un recaudo tributario como porcentaje del PIB del $13.73 \%$; siendo que, a partir de esta tasa el nivel de contribución marginal de los ingresos disminuye progresivamente según los postulados de Laffer.

Sin embargo, Oliva y Chiliquinga (2017) apunta a que la Curva de Laffer no debería se considerada por sí sola un modelo capaz de proyectar los niveles de recaudación tributaria, cuando es aceptado que éstos dependen de varios factores no incluidos en el modelo, tales como la estructura del sistema tributario, la temporalidad, el grado de informalidad del sistema, los posibles vacíos legales existentes, los factores cíclicos de la economía, entre otros; considerando al modelo como hiper reduccionista y generalmente analizado en un marco de análisis politizado.

De igual manera, autores como Oliva y Amoroso (2015) consideran a la Curva de Laffer como un argumento economicista basado en un modelo matemático abstracto que demuestra que una alta tasa de impuesto afecta a la recaudación dada una reducción en el crecimiento económico; siendo opinión de los autores que el modelo favorece a las grandes fortunas por ser ellos, precisamente, los creadores de la riqueza. Así, Falconí (2016) concluye que el concepto de la Curva de Laffer ha constituido un argumento empleado por los gobiernos neoliberales, a fin de justificar reducciones a los impuestos directos y sostener elevadas tasas de impuestos indirectos.

\section{Metodología}

Los datos empleados en el presente trabajo fueron tomados de las bases de datos de datos del Servicio de Rentas Internas (SRI), Banco Central (BCE)y del Instituto Ecuatoriano de Estadísticas y Censos (INEC), referentes a recaudación, Producto Interno Bruto y proyección poblacional respectivamente.

Inicialmente se muestra una descripción de la presión fiscal y recaudación percápita del periodo comprendido entre enero 2016 y diciembre 2017, expresado mediante medidas de tendencia central y dispersión.

En nuestro trabajo se utiliza la función cuadrática que especifica el modelo econométrico, representada por: 


$$
I T R_{t}=f\left(T t^{2}, T t\right)
$$

Siendo que $I T R_{t}$ representa los recaudos tributarios efectivos per cápita en el período $t$ y $T t$ representa los recaudos tributarios como porcentaje del Producto Interno Bruto. La forma funcional se plantea es planteada de la siguiente manera:

$$
I T R_{t}=\beta_{0}+\beta 1 T t+\beta 2 T t^{2}+\epsilon t
$$

Según anota Vidal, Caraguay y Salcedo (2017) en otros estudios propuestos, los modelos utilizan el IPC con el fin de eliminar las distorsiones por la inflación en el recaudo per cápita, más en el caso ecuatoriano se desecha esta variable y usas los recaudos a valor nominal, dados los bajos niveles de inflación en el período de análisis.

Es importante puntualizar que al analizar la presión tributaria en nuestro trajo relacionamos el total de impuestos efectivos recaudados por el SRI, dejando a un lado otros gravámenes tales como impuestos municipales, aranceles y pagas por seguridad social y otros; a fin de concentrar el análisis en los impuestos que se gestionan por parte del ente recaudador mencionado.

Para realizar las regresiones se aplicaron pruebas a los residuos de los modelos; la prueba de Breusch-Pagan para verificar la homocedasticidad, que hace referencia a que la varianza de los errores es constante a lo largo del tiempo; la prueba de Durbin Watson para determinar la autocorrelación identificada como la relación entre los valores de los residuos en un intervalo de tiempo y finalmente la prueba de normalidad para muestras pequeñas de Shapiro Wilk, en la que se supone que los datos están normalmente distribuidos.

Se realizaron las formas funcionales: lineal, logarítmica, lineal-logarítmica y logarítmica-lineal con y sin intercepto igual a cero; al encontrar la derivada de la ecuación cuadrática e igualando a cero se obtuvo la expresión: $-\beta_{1} / 2 \beta_{2}$, lo que determina el punto máximo de la curva que refleja el nivel óptimo. El procesamiento de los datos fue realizado mediante el programa estadístico SPSS 25 y la edición de tablas y gráficos en Excel 2016; las decisiones fueron tomadas con una consideración del $5 \%$. $(\mathrm{p}<0.05)$.

\section{Resultados}

Al realizar el análisis descriptivo se puede anotar que la recaudación fiscal, durante el periodo comprendido entre enero 2016 hasta diciembre 2017, tuvo oscilaciones entre el $4.7 \%$ (febrero 2016) y el $9.9 \%$ (abril 2017) con una media de $6.4 \%(\mathrm{DE}=1.1 \%)$; mientras que la presión fiscal durante el mismo periodo osciló entre el $9.3 \%$ (mayo 2016) y el $18.9 \%$ (mayo 2016) con una media de $12.7 \%$ (DE=2.5\%). En ambos casos se registraron bajas dispersiones de datos (CV $<20 \%$ ); lo que implica un comportamiento homogéneo durante los 24 meses.
La figura 1 muestra el comportamiento los recaudos tributarios efectivos per cápita $\left(I T R_{t}\right)$. y los recaudos tributarios como porcentaje del Producto Interno Bruto $(\mathrm{Tt})$. Se puede observar que de febrero del 2017 a diciembre del 2017 se mantuvo la recaudación per cápita con un promedio de $5.98 \%$, siendo que en el mes de abril se observa un incremento del $3.92 \%$ llegando a una recaudación del $9.9 \%$. Por otro lado, en los recaudos tributarios como porcentaje del PIB se encontró que durante el mismo periodo la media fue de $11.63 \%$ y que en el mes de abril este subió al $18.70 \%$ lo que indicó un incremento puntual de $7.07 \%$. Los picos encontrados se explican dado el hecho de que, por norma, las sociedades, pagan su IR en el mes de abril y al ser los mayores contribuyentes generan dicho efecto, Por otro lado, en el mes de enero se presente un pico de menor relevancia en cuanto al recaudo fiscal, el cual es consecuencia del pago del IVA generado por el auge comercial a efectos de las festividades navideñas.

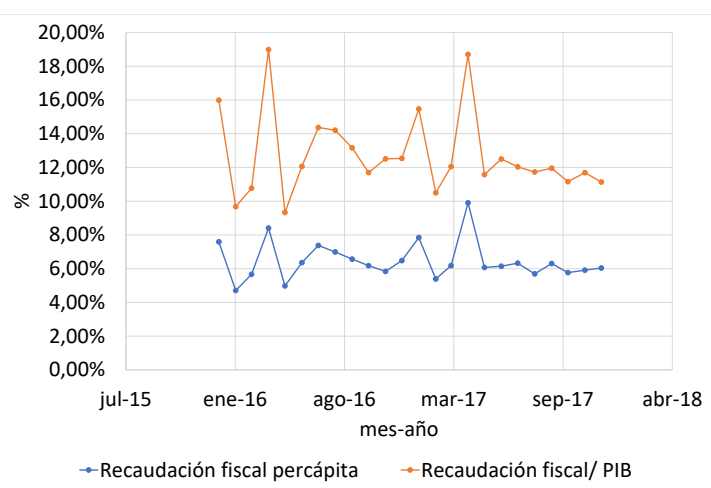

Figura 1. Tendencia presión fiscal per cápita y recaudación fiscal/PIB, 2016-2017

Fuente: (SRI, 2018) Estadísticas Generales de Recaudación. Obtenido de https://goo.gl/dSqo5E

Al verificar los supuestos en los modelos planteados, estos presentaron homocedasticidad, ausencia de autocorrelación y distribución normal, según los datos presentados en la Tabla 3, por ello se decidió aplicar el método de mínimos cuadrados.

Tabla 3 Resumen de pruebas aplicadas

\begin{tabular}{lcccc}
\hline Pruebas & $\begin{array}{c}\text { Forma } \\
\text { funcional }\end{array}$ & Estadístico & $\mathrm{p}$ & Condición \\
\hline $\begin{array}{l}\text { Prueba de normalidad (Sha- } \\
\text { piro Wilk) }\end{array}$ & Lin-Lin & 0,929 & 0,092 & Normal \\
& Log-Log & 0,950 & 0,274 & Normal \\
& Log-Lin & 0,928 & 0,087 & Normal \\
& Lin-Log & 0,955 & 0,341 & Normal \\
\hline $\begin{array}{l}\text { Prueba de Homcedasticidad } \\
\text { (Breusch-Pagan) }\end{array}$ & Lin-Lin & 0,929 & 0,000 & Homocedástico \\
& Log -Log & 0,950 & 0,034 & Homocedástico \\
& Log-Lin & 0,928 & 0,000 & Homocedástico \\
& Lin-Log & 0,955 & 0,069 & Heterocedástico \\
\hline
\end{tabular}


Tabla 3 Resumen de pruebas aplicadas (Continuación)

\begin{tabular}{lcccc}
\hline Pruebas & $\begin{array}{c}\text { Forma } \\
\text { funcional }\end{array}$ & Estadístico & $\mathrm{p}$ & Condición \\
\hline $\begin{array}{l}\text { Prueba de Autocorrelación } \\
\text { (Durbin Watson) }\end{array}$ & Lin-Lin & 2,102 & 0,000 & $\begin{array}{c}\text { Ausencia de } \\
\text { autocorrela- } \\
\text { ción }\end{array}$ \\
& Log-Log & 2,253 & 0,000 & $\begin{array}{c}\text { Ausencia de } \\
\text { autocorrela- } \\
\text { ción }\end{array}$ \\
& Log-Lin & 2,227 & 0,000 & $\begin{array}{c}\text { Ausencia de } \\
\text { autocorrela- } \\
\text { ción }\end{array}$ \\
& Lin-Log & 2,018 & 0,000 & $\begin{array}{c}\text { Ausencia de } \\
\text { autocorrela- } \\
\text { ción }\end{array}$ \\
\hline
\end{tabular}

La Tabla 4 muestra las formas funcionales estimadas para determinar la existencia de la curva de Laffer, en ella se observa que únicamente la función lineal respalda la curva, pues cumple con la condicionalidad de los signos del coeficiente para que la parábola tenga un sentido negativo y el intercepto es igual a cero pues no se descarga la posibilidad de considerar una una presión tributaria negativa.

Tabla 4 Índices de Ajuste del Modelo de Inteligencia emocional

\begin{tabular}{ccccccc}
\hline $\begin{array}{c}\text { Forma } \\
\text { funcional }\end{array}$ & Intercepto (p) & Tt (p) & Tt 2(p) & $\begin{array}{c}\text { R2 } \\
\text { ajustado }\end{array}$ & $\mathrm{p}$ & $\begin{array}{c}\text { Tasa } \\
\text { óptima } \\
(\%)\end{array}$ \\
\hline Lineal & 0 & 0,557 & $-0,406$ & 0,913 & 0,000 & n.a \\
& 0,984 & 0,057 & 0,678 & & & \\
\hline Log - $\log$ & $-0,58$ & 0,469 & $-0,237$ & 0,923 & 0,000 & n.a \\
& $-0,171$ & $-0,624$ & $-0,666$ & & & \\
\hline Log - lin & 0,276 & 0,338 & 0,114 & 0,913 & 0,000 & n.a \\
& $-0,001$ & $-0,046$ & $-0,225$ & & & \\
\hline Lin - Log & $-1,789$ & 6,292 & $-12,416$ & 0,922 & 0,000 & n.a \\
& 0 & $-0,001$ & $-0,043$ & & & \\
\hline Lin* & 0,562 & $-0,426$ & 0,998 & 0,000 & $65.96 \%$ \\
& $(0,000)$ & $-0,028$ & & & \\
\hline Log-Log* & 1,803 & 0,524 & 1,000 & 0,000 & n.a \\
& $(0,000)$ & $(0,000)$ & & & \\
\hline Log-Lin* & $-0,297$ & $-0,297$ & 0,996 & 0,000 & n.a \\
& $(0,000)$ & $(0,000)$ & & & \\
\hline Lin-Log* & $-19,428$ & 79,2 \\
& $(0,000)$ & $0,000)$ & & 0,998 & 0,000 & n.a \\
\hline
\end{tabular}

Nota: * En estos modelos, se omite el coeficiente (se restringe a que el intercepto sea igual a cero); n.a: No aplica

Luego, al proceder a maximizar la ecuación planteada mediante el empleo de la primera derivada de los recaudos fiscales per cápita con respeto a los recaudos tributarios como porcentaje del PIB, se encontró que la tasa que maximiza la curva planteada corresponde al $65.96 \%$ (ver punto B en la Figura 2), a partir de este nivel los incrementos en la presión fiscal provocaría una contracción de la recaudación per cápita.Cabe anotar que para diciembre de 2017 la relación recaudación fiscal PIB (presión tributaria) se estimó, según los datos obtenidos, en el $11.14 \%$ (ver punto A en la Figura 2), y una recaudación per cápita del $6.04 \%$. A continuación, se presentan los resultados de forma gráfica en la Figura 2:

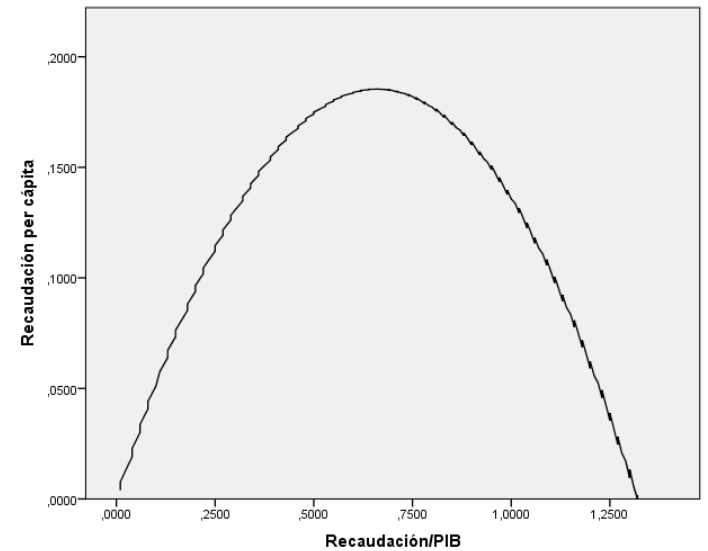

Figura 2. Simulación curva Laffer para el caso ecuatoriano años 2016 2017

Fuente: : elaboración propia

\section{Conclusiones}

Con el presente trabajo de investigación se pone en evidencia el cumplimiento de los parámetros especificados en la Curva de Laffer, poniéndose en evidencia la relación existente entre la presión tributaria y la recaudación tributaria per cápita.

A fin de demostrar la curva de Laffer se aplicó la función cuadrática según lo determina el modelo teórico, siendo que fueron estimadas las formas lineales, log-lin, lin-log y log-log, de las cuales la forma lineal presentó un mejor ajuste, siendo considerada ésta sin intercepto. Al evaluar el modelo en su forma lineal se apreció significancia estadística en los coeficientes de regresión, y por otro lado los signos son coincidentes con el marco teórico que establece Laffer.

Basados en los conceptos planteados, se estimó que el recaudo de impuestos en relación al PIB de $65.96 \%$ es el que maximiza los recaudos per cápita; siendo esta tasa la máxima recomendable aplicable luego de la cual los ingresos fiscales per cápita se ver reducidos. Por lo anotado, con el presente estudio se demuestra que la presión tributaria para el caso ecuatoriano está lejos de alcanzar sus niveles máximos, tomando como marco del análisis el modelo propuesto por Laffer.

Se puso en relieve los cambios al marco legal tributario durante el período de estudio, siendo evidente el crecimiento del recaudo tributario per cápita, tanto como de la relación recaudo PIB (presión tributaria). Se destaca la importancia del IVA en el sistema tributario ecuatoriano, que aun siendo un impuesto indirecto es el que más representa y el de mayor crecimiento en el período de análisis.

Es importante considerar la limitante de este trabajo al considerar como recaudo, en el análisis, únicamente los impuestos que están bajo el control del SRI, siendo importante tomar en cuenta que este estudio no considera los otros tributos ya detallados, a los cuales están sometidos los contribuyentes. Por otro lado, cabe subrayar que el 
crecimiento en el recaudo tributario en el período de estudio a más de ser el resultado de los cambios en el marco legal, se sustenta en el posible efecto de una eficiente gestión por parte del ente recaudador, por lo cual este trabajo constituye, únicamente, un estudio básico sobre el tema y una verificación empírica del modelo planteado.

\section{Referencias Bibliográficas}

Aliaga, J., y Oropeza, F. (2015). Análisis experimental de la curva de laffer y la evasión fiscal en bolivia. Revista Latinoamericana de Desarrollo Económico, 121-153.

Andes. (2018). SRI incrementa recaudación de Impuesto a la Renta en Ecuador. Autor.

Asamblea Nacional. (2016a). Ley Orgánica de Incentivos Tributarios para Varios Sectores Productivos e Interpretativa del Artículo 547 del Código Orgánico de Organización Territorial, Autonomía y Descentralización. Registro Oficial 860, 12-X-2016.

Asamblea Nacional. (2016b). Ley orgánica de solidaridad y de corresponsabilidad ciudadana para la reconstrucción y reactivación de las zonas afectadas por el terremoto de de abril de.

Asamblea Nacional. (2016c). Ley orgánica para evitar la especulación sobre el valor de las tierras y la fijación de tributos. Registro Oficial 913, 30-XII-2016.

Asamblea Nacional. (2017). Ley orgánica para la reactivación de la economía, fortalecimiento de la dolarización y modernización de la gestión financiera. Registro Oficial $N^{\circ} 150$ - Segundo Suplemento Viernes 29 de diciembre de 2017 - 3.

BCE. (2018). Ecuador creción $3.0 \%$ en 2017 y confirma el dinamismo de su economía.

Bejarano, H. (2008). Tributación en Colobia: La tasa óptima que maximiza el recaudo tributario. Revista Facultad de Ciencias Económicas: Investigación y Reflexión, 16(1), 151-164.

Casparri, M. (2013). La curva de Laffer y el impuesto inflacionario. (Tesis doctora). Facultad de Ciencias Económicas, Universidad de Buenos Aires.

Congreso Nacional. (2004). Ley Orgánico de Régimen Tributario Interno (Vol. Registro Oficial Suplemento 463 de 17-nov.-2004).

Cueva, P. (2012). Efectos de la tasa impositiva sobre los ingresos fiscales, mediante la aplicación de la Curva de Laffer, en la economía ecuatoriana (1970-2008). (Trabajo de grado). Universidad Técnica Particula de Loja, Loja, Ecuador.
Falconí, F. (2016). ¿Curva de Laffer o ley del embudo? El Telegráfo.

Gonzáles, D., y Doria, M. (2010). La curva de Laffer y la optimización del recaudo tributario en Cartagena Colombia. Revista Economía y Región.

Macías, H., y Cortés, J. (2004). Disminuri la tarifa general del IVA en Colombia aumentaría el recaudo tributario. Semestre económico, 7(13), 19-42.

Oliva, N., y Amoroso, X. (2015). Nueva política tributaria para el cambio de época posneoliberal. En Servicio de Rentas Interna, La nueva economía en la nueva Constitución del Ecuador (pp. 99-114). Quito, Ecuador.

Oliva, N., y Chiliquinga, D. (2017). La Curva de Laffer:. Propuestas para el desarrollo(1), 67-78.

Pérez, L., y Vaca, G. (2015). Impuesto al Valor Agregado (IVA): Evolución, tarifas y aportes de las principales ciudades: Caso Ecuador. Revista FENopina, 70.

Presidencia de la República. (2008). Reglamento para la aplicación del Impuesto a la Salida de Divisas. Registro Oficial 448.

SRI. (2018). Estadísticas Generales de Recaudación.

Varela, L., y Rubiera, F. (2017). Las aglomeraciones urbanas y los impuestos: algunas ideas derivadas de la aplicación de la curva de Laffer al impuesto sobre la renta español en diferentes escenarios espaciales. El trimestre económico, $\operatorname{LXXXIV(1),121-136.~}$

Vidal, F., Caraguay, M., y Salcedo, V. (2017). Carga tributaria y recaudación fiscal en el ecuador: un Análisis mediante la curva de laffer. Conference Proceedings, l(1).
Recibido: 30 de junio de 2018

Aceptado: 3 de septiembre de 2018 
